Research Paper

\title{
Integrating pre- and post-treatment Plasma Epstein-Barr Virus DNA levels for better prognostic prediction of Nasopharyngeal Carcinoma
}

\author{
Wanxia Li ${ }^{1,3 \#}$, Chao Yang4\#, Zehong Lv#, Junzheng $\mathrm{Li}^{6}$, Zonghua $\mathrm{Li}^{7}$, Xiaofei Yuan ${ }^{1}$, Shuting $\mathrm{Wu}^{1}$, Yue \\ Yuan ${ }^{1}$, Linchong Cui ${ }^{1}$, Juan Lu ${ }^{1 凶}$, Jing Chen ${ }^{5 凶}$ and Feipeng Zhao ${ }^{\circledR 凶}$ \\ 1. Department of Otolaryngology-Head and Neck Surgery, Nanfang Hospital, Southern Medical University, Guangzhou 510515, Guangdong, China. \\ 2. Department of Otolaryngology-Head and Neck Surgery, the Affiliated Hospital of Southwest Medical University, Southwest Medical University, Luzhou \\ 646000, Sichuan, China. \\ 3. Department of Health Management, Nanfang Hospital, Southern Medical University, Guangzhou 510515, Guangdong, China. \\ 4. Department of Laboratory Medicine, Nanfang Hospital, Southern Medical University, Guangzhou 510515, Guangdong, China. \\ 5. School of Traditional Chinese Medicine, Southern Medical University, Guangzhou 510515, Guangdong, China. \\ 6. Department of Otolaryngology-Head and Neck Surgery, Guangzhou Red Cross Hospital, Jinan University, Guangzhou 510220, Guangdong, China. \\ 7. Department of Otolaryngology, 942 Hospital of the Chinese People's Liberation Army, Yinchuan750001, Ningxia, China. \\ \#Co-first authors with equal contributions to this work.
}

$\square$ Corresponding authors: Juan Lu, Department of Otolaryngology-Head and Neck Surgery, Nanfang Hospital, Southern Medical University, Guangzhou 510515, China. Phone: 86-20-62787323; E-mail: lujuanqz@163.com. Jing Chen, School of Traditional Chinese Medicine, Southern Medical University, Guangzhou 510515, Guangdong, China. Phone: 86-20-61648771; E-mail: liuchengjing@126.com. Feipeng Zhao, Department of Otolaryngology-Head and Neck Surgery, the Affiliated Hospital of Southwest Medical University, Southwest Medical University, Luzhou 646000, China. Phone: 86-830-3165641; E-mail: zhaofeipeng008@swmu.edu.cn.

(c) The author(s). This is an open access article distributed under the terms of the Creative Commons Attribution License (https://creativecommons.org/licenses/by/4.0/). See http://ivyspring.com/terms for full terms and conditions.

Received: 2020.11.25; Accepted: 2021.02.03; Published: 2021.03.05

\begin{abstract}
Background: Pre- and post-treatment plasma Epstein-Barr virus (EBV) DNA are important biomarkers for the prognosis of nasopharyngeal carcinoma (NPC). This study was performed to determine the prognostic potential of integrating EBV DNA levels in plasma measured pre-treatment (pre-EBV) and 3 months post-treatment ( $3 \mathrm{~m}-\mathrm{EBV})$.

Materials and methods: A total of 543 incident non-metastatic NPC patients treated with intensitymodulated radiotherapy, with or without chemotherapy, were reviewed. Patients were divided into four subgroups based on pre-EBV and $3 \mathrm{~m}$-EBV status. The data for pre-EBV and $3 \mathrm{~m}-E B V$ samples were integrated, and the predictability of the survival of patients with NPC was analyzed.

Results: There were significant differences in the 5-year progression-free survival, distant metastasis-free survival, locoregional relapse-free survival, and overall survival among the four patient subgroups $(P<0.001)$. Patients who tested negative for both pre-EBV and $3 \mathrm{~m}$-EBV had the best prognosis, followed by patients who tested positive for pre-EBV and negative for $3 \mathrm{~m}-\mathrm{EBV}$, and those who tested negative for pre-EBV and positive for $3 \mathrm{~m}$-EBV; however, patients who tested positive for both pre-EBV and $3 \mathrm{~m}-E B V$ had the poorest chances of survival. Multivariate analyses demonstrated that integration of pre-EBV and $3 \mathrm{~m}-E B V$ data was an independent predictor of NPC progression in patients. Receiver operating characteristic curve analysis further confirmed that the combination of pre-EBV and $3 \mathrm{~m}$-EBV had a greater prognostic value than pre-EBV or $3 \mathrm{~m}-\mathrm{EBV}$ alone.

Conclusions: Integrating pre-EBV and $3 \mathrm{~m}$-EBV data could provide more accurate risk stratification and better prognostic prediction in NPC.
\end{abstract}

Key words: nasopharyngeal carcinoma; plasma; EBV DNA; prognosis; risk stratification

\section{Introduction}

Nasopharyngeal carcinoma (NPC) is endemic in Southeast Asia, especially in southern China, and has been established as an Epstein-Barr virus (EBV)- associated cancer [1]. Studies have demonstrated that EBV DNA in plasma originates from the tumor, and the load of plasma EBV DNA is strongly correlated 
with tumor burden [2]. In recent years, plasma EBV DNA has been widely used in clinical analysis as a reliable biomarker for screening, monitoring, and prognostic prediction of NPC [3-7]. A high pre-treatment plasma EBV DNA load correlates with advanced cancer stages, and poor prognosis [2, 8-11]. Conversely, detectable post-treatment EBV DNA levels are indicative of minimal residual disease and have been reported to be a stronger prognostic factor for NPC [2, 5, 12-15].

As pre- or post-treatment plasma EBV DNA is dynamic biomarkers [16, 17], combined evaluation of the changes in both may provide a more accurate prognosis. However, since most of the relevant studies mainly focused on prognosis with either preor post-treatment EBV DNA [18-21], to our knowledge, data on the investigation of the prognostic potential of simultaneous temporal changes in pre- and post-treatment plasma EBV DNA level for NPC are limited.

Therefore, we performed this retrospective study to evaluate the prognostic accuracy of the combination of plasma EBV DNA levels measured pre-treatment (pre-EBV) and 3 months post-treatment (3 m-EBV) for risk stratification and prognosis in NPC patients.

\section{Materials and Methods}

\section{Patients}

Data for a total of 543 patients with newly diagnosed, biopsy-proven, and nonmetastatic NPC treated at Nanfang Hospital, of Southern Medical University, from January 2008 to December 2015 were used in this study. Patients whose pre-EBV and/or 3 m-EBV data were not available were excluded. Patients with non-WHO pathological types, distant metastasis at primary diagnosis, and previous or other synchronous malignancies were also excluded. All patients were restaged according to the seventh edition of the American Joint Committee on Cancer (AJCC) staging system based on imaging materials and medical records [22]. Our retrospective study was approved by the Ethics Committee of Nanfang Hospital, Southern Medical University (NFEC-2017165).

\section{Treatment}

All patients were treated with 2.12-2.24 Gy per fraction, with five daily fractions per week, using intensity-modulated radiotherapy (IMRT) for a total of 6-8 weeks. Cumulative radiation doses were 70-74 Gy to the gross tumor target of the nasopharynx (GTVnx), 66-70 Gy to the neck metastatic lymph node area (GTVnd), 60-62 Gy to the high-risk clinical target volume (CTV1), and 50-56 Gy to the low-risk clinical target volume (CTV2). Concurrent chemotherapy (CCT) consisted of cisplatin, administered triweekly, or weekly until the end of radiotherapy. Induction or adjuvant chemotherapy (ICT/ACT) consisted of cisplatin with 5-fluorouracil or cisplatin with taxanes or all three used together, administered triweekly for 2 or 3 cycles. Among the patients, 32 (5.9\%) were at stage I and received IMRT treatment alone, 54 (9.9\%) were at stage II and received concurrent chemoradiotherapy (CCRT), and $457(84.2 \%)$ were at intermediate and advanced stages III/IV and received CCRT, ICT, and/or ACT.

\section{Follow-up and Endpoints}

All patients were routinely followed up every 3 months within the first year after therapy, every 6 months during the second and third years, and annually thereafter. Physical examination of the head and neck, nasopharyngeal endoscopy, MRI of the nasopharynx and neck, abdominal ultrasound, chest radiography, whole-body PET, and plasma EBV DNA measurements were performed routinely. PET/CT was considered if necessary.

The primary study endpoint was progressionfree survival (PFS), which was defined as the time from the initial pathological diagnosis of NPC to relapse at any site or death from any cause, whichever occurred first, or last follow-up visit. The secondary endpoints included distant metastasis-free survival (DMFS, distant metastasis detection, death, or last follow-up visit), locoregional relapse-free survival (LRFS, relapse in nasopharynx or neck lymph nodes, death, or last follow-up visit), and overall survival (OS, all-cause death or last follow-up visit).

\section{Quantification of plasma EBV DNA}

Plasma EBV DNA measurements were performed at pre-EBV and $3 \mathrm{~m}$-EBV stages using a real-time quantitative PCR technique targeting the BamH I-W region of the EBV genome. All plasma EBV DNA assays were conducted at the Laboratory Medicine Center of Nanfang Hospital, Southern Medical University. After the PCR assay, samples with an undetectable EBV DNA signal were recorded as 0 copies/mL, and a positive plasma EBV DNA load was defined as $>0$ copies $/ \mathrm{mL}$. Referring to previous studies $[2,6,7,23-26]$, the cutoff levels chosen to classify the patients into low and high EBV DNA groups were 1500 copies/mL pre-treatment and 0 copies/mL 3 months post-treatment in this study.

\section{Statistical analysis}

Survival outcomes were estimated using the Kaplan-Meier method and compared by the log-rank test. The Cox proportional hazard model was used for multivariate analysis including the following 
variables: sex, age ( $\geq 45$ vs. $<45$ years), $T$ stage ( $T_{4}$ vs. $\left.\mathrm{T}_{1-3}\right)$, $\mathrm{N}$ stage $\left(\mathrm{N}_{2-3} \mathrm{Vs} . \mathrm{N}_{0-1}\right)$, and the change in pre-EBV and $3 \mathrm{~m}$-EBV. ROC curve analysis was performed to calculate the optimal cut-off value of pre-EBV and 3 $\mathrm{m}-\mathrm{EBV}$, and compare the different prognostic values of pre-EBV, $3 \mathrm{~m}$-EBV, and the change in pre-EBV and $3 \mathrm{~m}$-EBV. Statistical analysis was performed using SPSS software version 21.0 (IBM Corporation, Armonk, NY, USA). Two-tailed $P$-values $<0.05$ were considered statistically significant.

\section{Results}

\section{Patient characteristics and survival outcomes}

Among the 543 NPC patients, 405 (74.6\%) were male and $138(25.4 \%)$ were female, the median age was 45.4 years (range: 13-75). Patient characteristics are listed in Table 1. During the median follow-up period of 49.2 months (range: 3-137 months), a total of 177 patients (32.6\%) experienced disease progression, including 44 cases of locoregional relapse $(8.1 \%), 95$ cases of distant metastasis (17.5\%), 24 cases of both locoregional relapse and distant metastasis $(4.4 \%)$, and 74 deaths $(13.6 \%, 60$ patients died from locoregional recurrence or distant metastasis and 14 patients died without locoregional recurrence or distant metastasis). The 5-year PFS, DMFS, LRFS, and OS rates were $66.1 \%, 75.6 \%, 86.0 \%$, and $83.8 \%$, respectively.

\section{Pre- and Post-treatment plasma EBV DNA assessment and survival outcomes}

Of the 543 patients, the positive rate $(61.0 \%)$ and median viral load $\left(926\right.$ copies $/ \mathrm{mL}$, range: $0-4.57 \times 10^{6}$ copies $/ \mathrm{mL}$ ) of pre-EBV samples were significantly higher than those for $3 \mathrm{~m}-\mathrm{EBV}(13.3 \%$ and 0 copies $/ \mathrm{mL}$, range: $0-1.16 \times 10^{7}$ copies $/ \mathrm{mL}$, respectively) (Figure 1A and 1B). Four different patterns were observed in pre-EBV and $3 \mathrm{~m}-\mathrm{EBV}$ (Figure 1C): (1) negative for both pre-EBV and 3 $\mathrm{m}-\mathrm{EBV}$; (2) positive for pre-EBV and negative for 3 $\mathrm{m}-\mathrm{EBV}$; (3) negative for pre-EBV and positive for 3 $\mathrm{m}-\mathrm{EBV}$; (4) positive for both pre-EBV and $3 \mathrm{~m}$-EBV.
Also, the entire cohort of 543 patients was divided into two groups based on plasma EBV DNA cut-off values of 1500 copies $/ \mathrm{ml}$ for pre-EBV and 0 copies $/ \mathrm{ml}$ for $3 \mathrm{~m}$-EBV. The results of survival analysis showed that patients with pre-EBV load $\geq 1500$ copies/mL had worse 5-year PFS, DMFS, LRFS, and OS than those with $<1500$ copies/mL (all $P<$ 0.001). Similarly, the 5-year PFS, DMFS, LRFS, and OS were significantly lower among patients with positive $(>0$ copies $/ \mathrm{mL}) 3 \mathrm{~m}$-EBV than in patients with negative plasma EBV DNA (all $P<0.001$ ). KaplanMeier survival curves for survival analyses of subgroups are shown in Figure 2.

Table 1. Clinical characteristics of NPC patients $(n=543)$

\begin{tabular}{|c|c|}
\hline Characteristic & $\mathrm{N}(\%)$ \\
\hline \multicolumn{2}{|l|}{ Sex } \\
\hline Female & $138(25.4 \%)$ \\
\hline Male & $405(74.6 \%)$ \\
\hline \multicolumn{2}{|l|}{ Age (years) } \\
\hline$<45$ & $252(46.4 \%)$ \\
\hline$\geq 45$ & $291(53.6 \%)$ \\
\hline \multicolumn{2}{|l|}{ Smoking } \\
\hline Yes & $322(49.3 \%)$ \\
\hline No & $221(40.7 \%)$ \\
\hline \multicolumn{2}{|l|}{ WHO pathologic type ${ }^{1}$} \\
\hline Keratinizing carcinoma & $3(0.6 \%)$ \\
\hline Differentiated non-keratinizing carcinoma & $34(6.3 \%)$ \\
\hline Undifferentiated non-keratinizing carcinoma & $507(93.1 \%)$ \\
\hline \multicolumn{2}{|l|}{ Overall stage $^{2}$} \\
\hline I & $32(5.9 \%)$ \\
\hline II & $54(9.9 \%)$ \\
\hline III & $186(34.3 \%)$ \\
\hline IV & $271(49.9 \%)$ \\
\hline \multicolumn{2}{|l|}{ Tumor stage $^{2}$} \\
\hline $\mathrm{T}_{1}$ & $99(18.2 \%)$ \\
\hline $\mathrm{T}_{2}$ & $92(16.9 \%)$ \\
\hline $\mathrm{T}_{3}$ & $109(20.1 \%)$ \\
\hline $\mathrm{T}_{4}$ & $243(44.8 \%)$ \\
\hline \multicolumn{2}{|l|}{ Node stage $^{2}$} \\
\hline $\mathrm{N}_{0}$ & $59(10.9 \%)$ \\
\hline $\mathrm{N}_{1}$ & $144(26.5 \%)$ \\
\hline $\mathrm{N}_{2}$ & $295(54.3 \%)$ \\
\hline $\mathrm{N}_{3}$ & $45(8.3 \%)$ \\
\hline
\end{tabular}

${ }^{1}$ Pathologic type according to the 2005 World Health Organization (WHO) classification of tumors.

${ }^{2}$ According to the $7^{\text {th }}$ edition of the AJCC staging system.
A

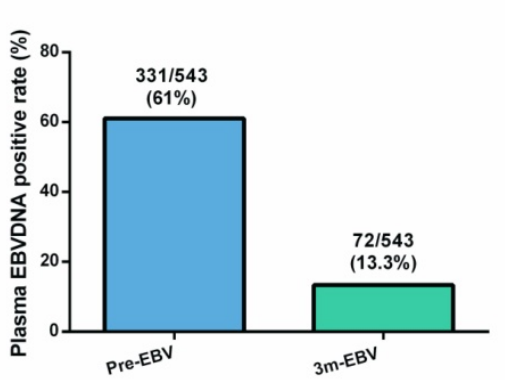

B

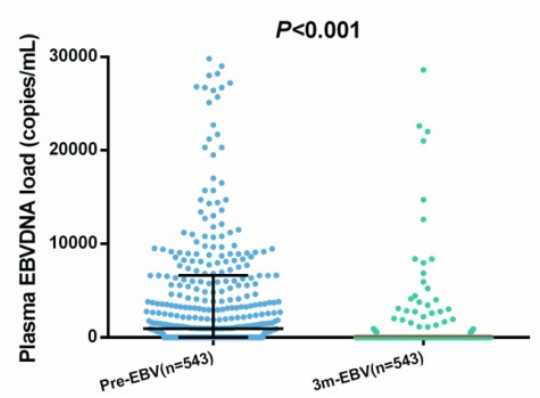

C

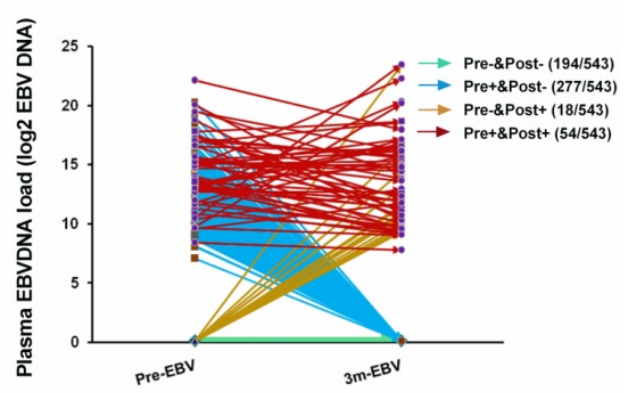

Figure 1. The positive rate and viral load of pre-treatment (Pre-EBV) and 3 months post-treatment ( $3 \mathrm{~m}$-EBV) plasma EBV DNA. (A) Comparisons of the positive rates for Pre-EBV and $3 \mathrm{~m}-\mathrm{EBV}$ values. (B) Comparisons of plasma Pre-EBV and $3 \mathrm{~m}-\mathrm{EBV}$ values. (C) The changes in Pre-EBV and $3 \mathrm{~m}-\mathrm{EBV}$ DNA levels in plasma. 
A

B

DMFS
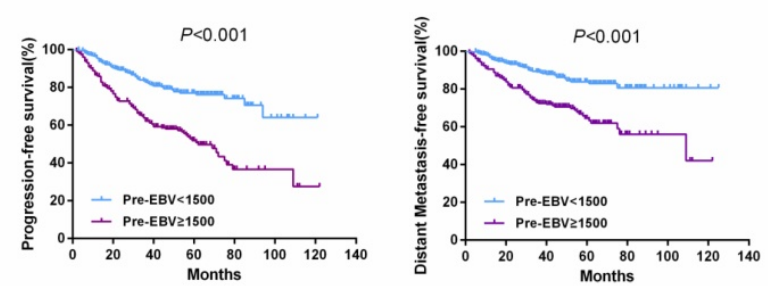

C

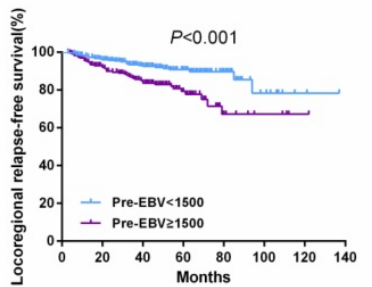

D

OS

Pre-EBV

$3 m-E B V$
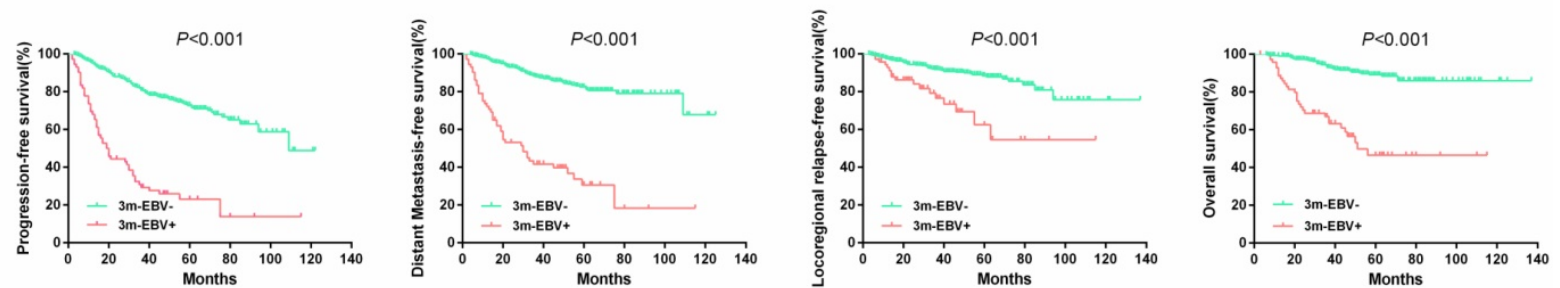

Figure 2. Kaplan-Meier curves of survival outcomes among subgroups defined by pre-treatment (Pre-EBV) and 3 months post-treatment (3 m-EBV) plasma EBV DNA levels. (A) Progression-free survival (PFS). (B) Distant metastasis-free survival (DMFS). (C) Locoregional relapse-free survival (LRFS). (D) Overall survival (OS).

A

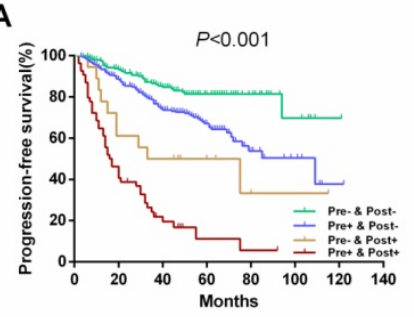

B

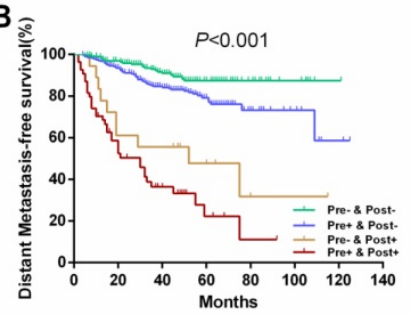

E

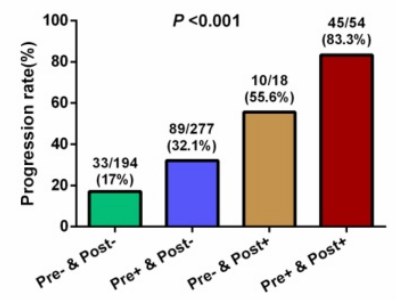

$\mathbf{F}$

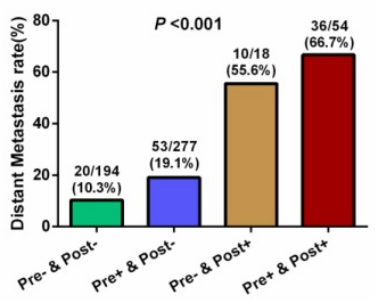

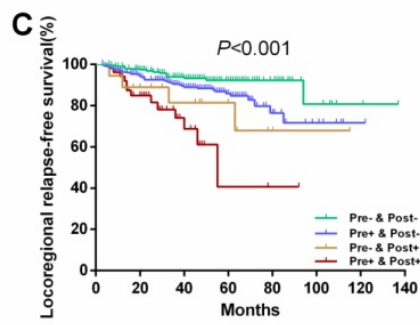

D

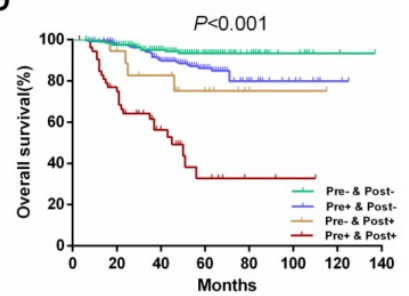

H

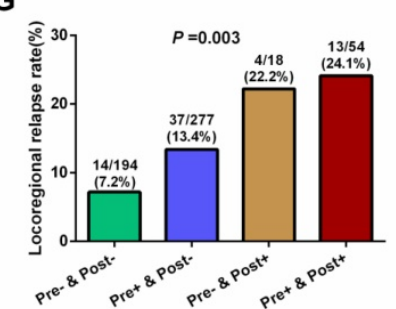

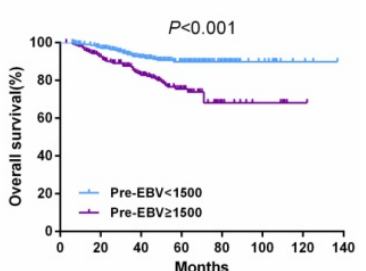

Figure 3. Survival outcomes and treatment failure rates among four patient subgroups according to the combinations of pre- and 3 months post-treatment plasma EBV DNA levels. (A) Progression-free survival. (B) Distant metastasis-free survival. (C) Locoregional relapse-free survival. (D) Overall survival. (E) Progression rate. (F) Distant metastasis rate. (G) Locoregional relapse rate. (H) Mortality rate. "Pre-" and "Pre+" denote negative and positive pre-treatment EBV DNA status, respectively; "Post-" and "Post+" denote negative and positive 3 months post-treatment EBV DNA status, respectively.

\section{Combination of Pre-EBV and 3 m-EBV data}

As the aforementioned analyses showed, both pre-EBV and $3 \mathrm{~m}$-EBV data were effective prognostic factors for NPC patients. Therefore, we stratified the entire population into four subgroups according to the change in the two prognostic factors for pre-EBV and $3 \mathrm{~m}-\mathrm{EBV}$ : negative for both pre-EBV and $3 \mathrm{~m}-\mathrm{EBV}$ (Group 1: Pre- and Post-, n=194); positive for pre-EBV and negative for $3 \mathrm{~m}-\mathrm{EBV}$ (Group 2: Pre+ and Post-, $\mathrm{n}=277$ ); negative for pre-EBV and positive for 3 m-EBV (Group 3: Pre- and Post+, $\mathrm{n}=18$ ); positive for both pre-EBV and $3 \mathrm{~m}$-EBV (Group 4: Pre+ and Post+, n=54) (Table 2).

Results of further subgroup prognostic analyses are presented in Figure 3. Differences for 5-year PFS $(81.4 \%, 67.1 \%, 50.0 \%$, and $11.2 \%$ for Groups 1 to 4 , respectively), DMFS $(87.3 \%, 79.2 \%, 47.6 \%$, and $22.2 \%$ for Groups 1 to 4, respectively), LRFS (92.3\%, 85.9\%, $81.5 \%$, and $40.7 \%$ for Groups 1 to 4 , respectively), and OS $(93.5 \%, 86.2 \%, 75.1 \%$, and $32.8 \%$ for Groups 1 to 4 , respectively) were statistically significant among the above four subgroups (all $P<0.001$; Figure 3A-D). Similarly, the disease progression, distant metastasis, locoregional relapse, and mortality rates were significant among these four subgroups (all $P<0.05$; 
Figure 3E-H). Patients who tested negative for both pre-EBV and $3 \mathrm{~m}$-EBV had the best prognosis, followed by patients who tested positive for pre-EBV and negative for $3 \mathrm{~m}-\mathrm{EBV}$, and negative for pre-EBV and positive for $3 \mathrm{~m}$-EBV subgroup patients; patients who tested positive for both pre-EBV and $3 \mathrm{~m}-\mathrm{EBV}$ had the poorest survival outcomes.

Table 2. Subgroups of the change in plasma EBV DNA levels pre-treatment (pre-EBV) and 3 months post-treatment ( $3 \mathrm{~m}$-EBV)

\begin{tabular}{llll}
\hline Timepoints & & $3 \mathrm{~m}$-EBV & \\
\cline { 3 - 4 } & & Negative & Positive \\
\hline Pre-EBV & Negative & $194(35.7 \%)$ & $18(3.3 \%)$ \\
& Positive & $277(51.0 \%)$ & $54(10.0 \%)$ \\
\hline
\end{tabular}

\section{Cox multivariate analysis}

Multivariate analysis revealed that integrating pre-EBV and $3 \mathrm{~m}$-EBV plasma EBV DNA status could be employed as an independent predictor of PFS, DMFS, LRFS, and OS in NPC patients. Compared with the "Pre- and Post-" subgroup, the "Pre- and Post+" and "Pre+ and Post + " subgroups were independent risk factors for worse PFS, DMFS, LRFS, and OS (all $P<0.001$ ), whereas the "Pre+ and Post-" subgroup was an independent risk factor for comparatively poorer PFS $(P=0.002)$ and DMFS $(P=0.031)$ (Table 3).

Further, the "Pre+ and Post+" subgroup patients had a significantly higher risk of disease progression (hazard ratio (HR), 9.678; 95\% confidence interval (CI), 6.019-15.559), distant metastasis (HR, 10.488; 95\% CI, 5.924-18.568), locoregional recurrence (HR, 5.628; 95\% CI, 2.553-12.404), and death (HR, 11.587; 95\% CI, 5.576-24.080) than the "Pre- and Post-" group (Table $3)$.

\section{ROC curve analysis}

By comparing the ROC curves, integrating the pre-EBV and $3 \mathrm{~m}$-EBV status demonstrated larger area under the curve (AUC) values than pre-EBV or 3 $\mathrm{m}-\mathrm{EBV}$ alone for predicting NPC progression (AUC $=0.697 ; \quad P<0.001$ ), distant metastasis (AUC $=0.711 ; \quad P<0.001$ ), locoregional relapse $(\mathrm{AUC}=0.618 ; P=0.002)$, and mortality $(\mathrm{AUC}=0.710 ; P<$ 0.001) (Figure 4).

\section{Discussion}

In the past two decades, plasma EBV DNA, an archetypal circulating tumor DNA, has been recognized as a robust biomarker for NPC [27]. Previous studies have confirmed that pre- and post-treatment plasma EBV DNA have independent prognostic value in patients with NPC $[2,5-15,28,29]$. The above findings have been further confirmed in our research. We also found that there were significant changes in the positive rate and load of plasma EBV DNA after treatment, and these findings led us to investigate whether the integrated pre-EBV and $3 \mathrm{~m}$-EBV data improve prognostic stratification for NPC patients.

To the best of our knowledge, the performance of the combination of pre- and post-treatment plasma EBV DNA for predicting treatment failure in NPC patients has not been fully investigated, partly because of the limited availability of data and the lack of comprehensive subgroup analyses. In this study, we used the long-term follow-up clinical database with a large sample size, and all eligible patients were divided into four subgroups. We found that patients with persistently negative pre-EBV and $3 \mathrm{~m}$-EBV had the best survival outcome, while patients with consistently positive pre-EBV and $3 \mathrm{~m}$-EBV had the worst prognosis. This result is consistent with previous studies $[12,23]$.

Furthermore, by using the multivariate prognostic model, our data also showed that patients with consistently positive pre-EBV and $3 \mathrm{~m}$-EBV had a significantly higher risk of disease progression, distant metastasis, locoregional recurrence, and death than the persistently negative group (Table 3 ). The most reasonable explanation for the poor prognosis of these patients is that they either had uncontrolled tumors, unfavorable treatment responses, or residual diseases, which may have progressed with high risk $[5,30]$. Moreover, closer follow-up visits and further intensified therapy or timely salvage treatment might be beneficial for these high-risk subgroup patients, while excessive or non-contributive treatment and examination can be avoided for patients with persistently negative EBV DNA [31, 32].

Another important finding from our study was that the "Pre- and Post+" patients were revealed to have worse 5-year PFS, DMFS, LRFS, and OS than the "Pre- and Post-", as well as "Pre+ and Post-" patients. Notably, the prognosis of this subgroup of patients remains controversial, mainly because of a limited number of previous studies, carried out with a small sample size of patients [11, 13, 17]. Although our results differ from those obtained by Peng et al. [11], they are broadly consistent with the findings obtained by Lin et al. [23] and Li et al. [13]. EBV DNA can be detected in NPC tumor cells [33]. Also, cell-free EBV DNA can be detected in the plasma of patients with NPC, which may come from necrosis and lysis of tumor cells infected with EBV [34, 35]. In our study, 18 patients $(8.5 \%)$ with positive $3 \mathrm{~m}$-EBV were found among the 212 patients with negative pre-EBV. It may be that EBV infection has occurred in the nasopharyngeal carcinoma tissue of NPC patients in the "Pre- and Post+" group before treatment, but EBV 
DNA could not be detected in the plasma. Next, we will collect nasopharyngeal tumor tissues and venous blood samples of NPC patients in the "Pre- and Post+" group before and after treatment, and detect EBV DNA in tumor cells and plasma to confirm this possibility.

Additionally, ROC curve analysis further confirmed that integrating pre-EBV and $3 \mathrm{~m}-\mathrm{EBV}$ had greater prognostic value than pre-EBV or $3 \mathrm{~m}-\mathrm{EBV}$ analyzed alone, which was similar to the findings obtained by Peng et al. [11]. Considering the above results, integrating pre-EBV and $3 \mathrm{~m}-\mathrm{EBV}$ data yielded a strong independent prognostic factor for

A

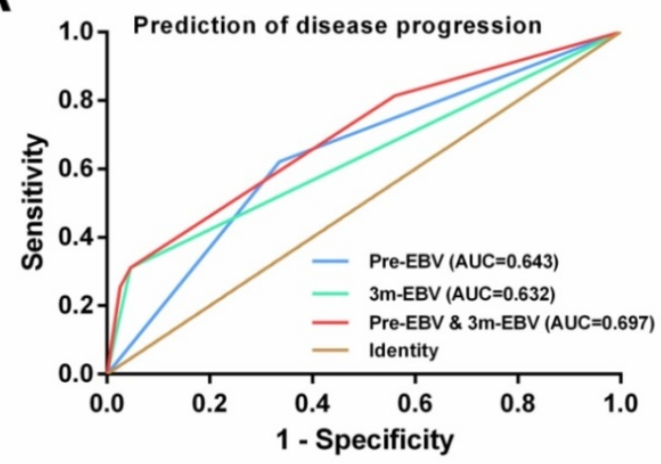

C

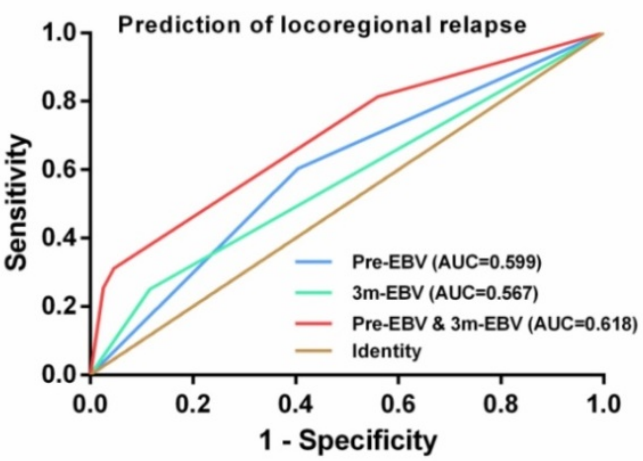

NPC patients, highlighting the feasibility and clinical application in NPC prognostic stratification and tumor surveillance post-treatment.

Our study had some limitations as well. First, some potential biases were unavoidable owing to the retrospective design. Second, the population enrolled in the study was considerably small, especially the sample size for the "Pre- and Post+" subgroup. Moreover, all NPC patients originated from one center and there was no validation cohort. Therefore, larger sample-sized, prospective, multi-center, randomized, and controlled clinical studies are required to further validate our findings.

B

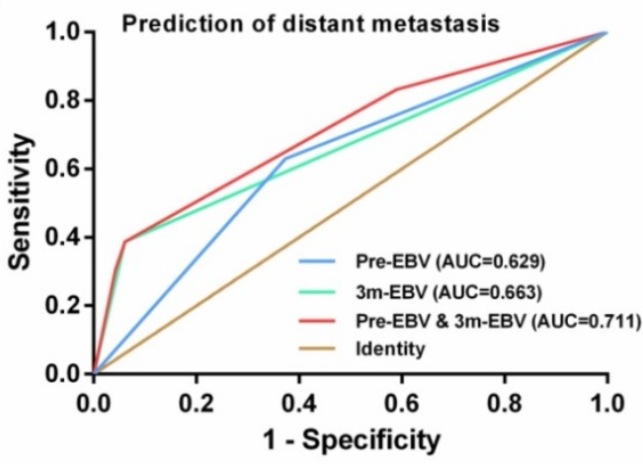

D

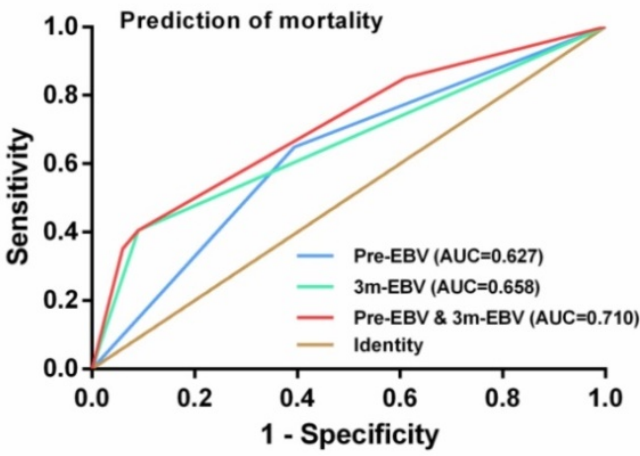

Figure 4. ROC curve analysis for comparing the prognostic potential of pre-treatment (pre-EBV), 3 months post-treatment (3 m-EBV) plasma EBV DNA levels, and integrated pre-EBV and $3 \mathrm{~m}-\mathrm{EBV}$ values. (A) Prediction of disease progression. (B) Prediction of distant metastasis. (C) Prediction of locoregional relapse. (D) Prediction of mortality. AUC: the area under the curve.

Table 3. Multivariate analysis of prognostic factors of NPC patients

\begin{tabular}{|c|c|c|c|c|c|c|c|c|}
\hline \multirow[t]{2}{*}{ Variable } & \multicolumn{2}{|l|}{ PFS } & \multicolumn{2}{|l|}{ DMFS } & \multicolumn{2}{|l|}{ LRFS } & \multicolumn{2}{|l|}{ OS } \\
\hline & HR (95\% CI) & $P$ value & HR (95\% CI) & $P$ value & HR $(95 \% \mathrm{CI})$ & $P$ value & HR $(95 \%$ CI) & $P$ value \\
\hline Sex (male vs. female) & $1.273(0.856-1.895)$ & 0.233 & $1.296(0.798-2.105)$ & 0.294 & $0.890(0.477-1.661)$ & 0.714 & $1.532(0.805-2.916)$ & 0.194 \\
\hline Age ( $\geq 45$ vs. $<45)$ & $1.151(0.852-1.555)$ & 0.360 & $1.063(0.736-1.536)$ & 0.744 & $1.079(0.664-1.754)$ & 0.759 & $1.718(1.054-2.798)$ & 0.030 \\
\hline Smoking (Yes vs. No) & $0.996(0.706-1.404)$ & 0.981 & $0.983(0.644-1.500)$ & 0.937 & $1.171(0.666-2.061)$ & 0.584 & $1.000(0.592-1.687)$ & 0.999 \\
\hline $\mathrm{T}$ stage $\left(\mathrm{T}_{4}\right.$ vs. $\left.\mathrm{T}_{1-3}\right)$ & $1.163(0.859-1.574)$ & 0.329 & $1.023(0.705-1.484)$ & 0.905 & $1.661(1.013-2.724)$ & 0.044 & $1.122(0.700-1.799)$ & 0.633 \\
\hline $\mathrm{N}$ stage $\left(\mathrm{N}_{2-3}\right.$ vs. $\left.\mathrm{N}_{0-1}\right)$ & $1.256(0.900-1.753)$ & 0.180 & $1.646(1.071-2.531)$ & 0.023 & $1.024(0.612-1.716)$ & 0.927 & $1.702(0.988-2.931)$ & 0.055 \\
\hline \multicolumn{2}{|c|}{ EBV DNA change subgroup } & $<0.001$ & & $<0.001$ & & $<0.001$ & & $<0.001$ \\
\hline Pre- \& Post- & Reference & Reference & Reference & Reference & Reference & Reference & Reference & Reference \\
\hline Pre+ \& Post- & $1.878(1.252-2.818)$ & 0.002 & $1.773(1.053-2.983)$ & 0.031 & $1.814(0.970-3.389)$ & 0.062 & $1.912(0.959-3.812)$ & 0.066 \\
\hline Pre- \& Post+ & 4.167 (2.032-8.547) & $<0.001$ & $7.254(3.338-15.762)$ & $<0.001$ & $3.388(1.104-10.400)$ & 0.033 & $4.582(1.442-14.554)$ & 0.010 \\
\hline Pre+ \& Post+ & $9.678(6.019-15.559)$ & $<0.001$ & $10.488(5.924-18.568)$ & $<0.001$ & $5.628(2.553-12.404)$ & $<0.001$ & $11.587(5.576-24.080)$ & $<0.001$ \\
\hline
\end{tabular}

Abbreviations: PFS: progression-free survival; DMFS: distant metastasis-free survival; LRFS: locoregional relapse-free survival; OS: overall survival; HR: hazard ratio; CI: confidence interval. 


\section{Conclusions}

In summary, the results of the current study demonstrated that integrating pre-EBV and $3 \mathrm{~m}-\mathrm{EBV}$ data was an effective prognostic predictor for NPC patients, which could further provide more accurate risk stratification. Our study may help guide individual management for NPC patients' in future clinical practice. However, further studies with larger sample sizes and multiple patient origins will be helpful in establishing the reliability of the proposed method.

\section{Abbreviations}

3 m-EBV: 3 months post-treatment; AUC: area under the curve; CTV1: clinical target volume; CCRT: concurrent chemoradiotherapy; CCT: Concurrent chemotherapy; CI: confidence interval; DMFS: distant metastasis-free survival; EBV: Epstein-Barr virus; GTVnx: gross tumor target of the nasopharynx; ICT/ACT: Induction or adjuvant chemotherapy; IMRT: intensity-modulated radiotherapy; LRFS: locoregional relapse-free survival; CTV2: low-risk clinical target volume; NPC: nasopharyngeal carcinoma; GTVnd: gross tumor target of neck metastatic lymph node area; OS: overall survival; pre-EBV: pre-treatment; PFS: progression-free survival; ROC: receiver operator characteristic.

\section{Acknowledgements}

This work was supported by grants from the National Natural Science Foundation of China (81602570) and the Natural Science Foundation of Guangdong Province (2020A1515010596 and 2018A030313572).

\section{Availability of data and material}

The data that support the findings of this study are available from the corresponding author upon reasonable request.

\section{Author Contributions}

Study concept: Feipeng Zhao. Study design: Juan Lu and Jing Chen. Data acquisition: Wanxia Li, Chao Yang, and Zehong Lv. Quality control of data and algorithms: Yue Yuan and Linchong Cui. Data analysis and interpretation: Junzheng $\mathrm{Li}$, Zonghua $\mathrm{Li}$, Xiaofei Yuan, and Shuting Wu. Statistical analysis: Junzheng Li, Zonghua $\mathrm{Li}$, and Xiaofei Yuan. Article preparation: Wanxia Li, Chao Yang, and Zehong Lv. Article editing: Juan Lu and Jing Chen. Article review: All authors.

\section{Competing Interests}

The authors have declared that no competing interest exists.

\section{References}

1. Chen YP, Chan ATC, Le QT, Blanchard P, Sun Y, Ma J. Nasopharyngeal carcinoma. Lancet 2019, 394(10192):64-80.

2. Lin JC, Wang WY, Chen KY, Wei YH, Liang WM, Jan JS, Jiang RS, Quantification of plasma Epstein-Barr virus DNA in patients with advanced nasopharyngeal carcinoma. N Engl J Med 2004, 350(24):2461-2470.

3. Chan KCA, Woo JKS, King A, Zee BCY, Lam WKJ, Chan SL, Chu SWI, Mak C, Tse IOL, Leung SYM, et al. Analysis of Plasma Epstein-Barr Virus DNA to Screen for Nasopharyngeal Cancer. N Engl J Med 2017, 377(6):513-522.

4. Lo YM, Chan AT, Chan LY, Leung SF, Lam CW, Huang DP, Johnson PJ. Molecular prognostication of nasopharyngeal carcinoma by quantitative analysis of circulating Epstein-Barr virus DNA. Cancer Res 2000, 60(24):6878-6881

5. Chan AT, Lo YM, Zee B, Chan LY, Ma BB, Leung SF, Mo F, Lai M, Ho S, Huang DP, et al. Plasma Epstein-Barr virus DNA and residual disease after radiotherapy for undifferentiated nasopharyngeal carcinoma. J Natl Cancer Inst 2002, 94(21):1614-1619.

6. Wang WY, Twu CW, Chen HH, Jiang RS, Wu CT, Liang KL, Shih YT, Chen CC, Lin PJ, Liu YC, et al. Long-term survival analysis of nasopharyngeal carcinoma by plasma Epstein-Barr virus DNA levels. Cancer 2013, 119(5):963-970.

7. Zhao FP, Liu X, Chen XM, Lu J, Yu BL, Tian WD, Wang LU, Xu X, Huang HR, Zhang MW, et al. Levels of plasma Epstein-Barr virus DNA prior and subsequent to treatment predicts the prognosis of nasopharyngeal carcinoma. Oncol Lett 2015, 10(5):2888-2894.

8. Lo YM, Leung SF, Chan LY, Lo KW, Zhang J, Chan AT, Lee JC, Hjelm NM, Johnson PJ, Huang DP. Plasma cell-free Epstein-Barr virus DNA quantitation in patients with nasopharyngeal carcinoma. Correlation with clinical staging. Ann N Y Acad Sci 2000, 906:99-101.

9. Leung SF, Zee B, Ma BB, Hui EP, Mo F, Lai M, Chan KC, Chan LY, Kwan WH, Lo YM, et al. Plasma Epstein-Barr viral deoxyribonucleic acid quantitation complements tumor-node-metastasis staging prognostication in nasopharyngeal carcinoma. J Clin Oncol 2006, 24(34):5414-5418.

10. Guo R, Tang LL, Mao YP, Du XJ, Chen L, Zhang ZC, Liu LZ, Tian L, Luo XT, $\mathrm{Xie} Y \mathrm{Y}$, et al. Proposed modifications and incorporation of plasma Epstein-Barr virus DNA improve the TNM staging system for Epstein-Barr virus-related nasopharyngeal carcinoma. Cancer 2019, 125(1):79-89.

11. Peng H, Guo R, Chen L, Zhang Y, Li WF, Mao YP, Sun Y, Zhang F, Liu LZ, Lin $\mathrm{AH}$, et al. Prognostic Impact of Plasma Epstein-Barr Virus DNA in Patients with Nasopharyngeal Carcinoma Treated using Intensity-Modulated Radiation Therapy. Sci Rep 2016, 6:22000.

12. Lo YM, Chan LY, Chan AT, Leung SF, Lo KW, Zhang J, Lee JC, Hjelm NM, Johnson PJ, Huang DP. Quantitative and temporal correlation between circulating cell-free Epstein-Barr virus DNA and tumor recurrence in nasopharyngeal carcinoma. Cancer Res 1999, 59(21):5452-5455.

13. Li WF, Zhang Y, Huang XB, Du XJ, Tang LL, Chen L, Peng H, Guo R, Sun Y, Ma J. Prognostic value of plasma Epstein-Barr virus DNA level during posttreatment follow-up in the patients with nasopharyngeal carcinoma having undergone intensity-modulated radiotherapy. Chin J Cancer 2017, 36(1):87

14. Le QT, Jones CD, Yau TK, Shirazi HA, Wong PH, Thomas EN, Patterson BK, Lee AW, Zehnder JL. A comparison study of different PCR assays in measuring circulating plasma epstein-barr virus DNA levels in patients with nasopharyngeal carcinoma. Clin Cancer Res 2005, 11(16):5700-5707.

15. Hou X, Zhao C, Guo Y, Han F, Lu LX, Wu SX, Li S, Huang PY, Huang H, Zhang L. Different clinical significance of pre- and post-treatment plasma Epstein-Barr virus DNA load in nasopharyngeal carcinoma treated with radiotherapy. Clin Oncol (R Coll Radiol) 2011, 23(2):128-133.

16. He SS, Wang $Y$, Bao $Y$, Cai XY, Yang XL, Chen DM, Chen $Y$, Lu LX. Dynamic changes in plasma Epstein-Barr virus DNA load during treatment have prognostic value in nasopharyngeal carcinoma: a retrospective study. Cancer Med 2018, 7(4):1110-1117.

17. Prayongrat A, Chakkabat C, Kannarunimit D, Hansasuta P, Lertbutsayanukul C. Prevalence and significance of plasma Epstein-Barr Virus DNA level in nasopharyngeal carcinoma. J Radiat Res 2017, 58(4):509-516.

18. Chen YP, Zhao BC, Chen C, Shen LJ, Gao J, Mai ZY, Chen MK, Chen G, Yan F, Liu S, et al. Pretreatment platelet count improves the prognostic performance of the TNM staging system and aids in planning therapeutic regimens for nasopharyngeal carcinoma: a single-institutional study of 2,626 patients. Chin J Cancer 2015, 34(3):137-146.

19. Lee VH, Kwong DL, Leung TW, Choi CW, O'Sullivan B, Lam KO, Lai V, Khong PL, Chan SK, Ng CY, et al. The addition of pretreatment plasma Epstein-Barr virus DNA into the eighth edition of nasopharyngeal cancer TNM stage classification. Int J Cancer 2019, 144(7):1713-1722.

20. Chan ATC, Hui EP, Ngan RKC, Tung SY, Cheng ACK, Ng WT, Lee VHF, Ma BBY, Cheng HC, Wong FCS, et al. Analysis of Plasma Epstein-Barr Virus DNA in Nasopharyngeal Cancer After Chemoradiation to Identify High-Risk Patients for Adjuvant Chemotherapy: A Randomized Controlled Trial. J Clin Oncol 2018:JCO2018777847.

21. Hui EP, Li WF, Ma BB, Lam WKJ, Chan KCA, Mo F, Ai QYH, King AD, Wong $\mathrm{CH}$, Guo R, et al. Integrating postradiotherapy plasma Epstein-Barr virus 
DNA and TNM stage for risk stratification of nasopharyngeal carcinoma to adjuvant therapy. Ann Oncol 2020.

22. Edge SB, Compton CC. The American Joint Committee on Cancer: the 7th edition of the AJCC cancer staging manual and the future of TNM. Ann Surg Oncol 2010, 17(6):1471-1474.

23. Lin JC, Wang WY, Liang WM, Chou HY, Jan JS, Jiang RS, Wang JY, Twu CW, Liang KL, Chao J, et al. Long-term prognostic effects of plasma epstein-barr virus DNA by minor groove binder-probe real-time quantitative PCR on nasopharyngeal carcinoma patients receiving concurrent chemoradiotherapy. Int J Radiat Oncol Biol Phys 2007, 68(5):1342-1348.

24. Wei W, Huang Z, Li S, Chen H, Zhang G, Li S, Hu W, Xu T. Pretreatment Epstein-Barr virus DNA load and cumulative cisplatin dose intensity affect long-term outcome of nasopharyngeal carcinoma treated with concurrent chemotherapy: experience of an institute in an endemic area. Oncol Res Treat 2014, 37(3):88-95.

25. Jin YN, Yao JJ, Zhang F, Wang SY, Zhang WJ, Zhou GQ, Qi ZY, Sun Y. Is pretreatment Epstein-Barr virus DNA still associated with 6-year survival outcomes in locoregionally advanced nasopharyngeal carcinoma? J Cancer 2017, 8(6):976-982

26. Chen QY, Tang QN, Tang LQ, Chen WH, Guo SS, Liu LT, Li CF, Li Y, Liang YJ, Sun XS, et al. Pretreatment Serum Amyloid A and C-reactive Protein Comparing with Epstein-Barr Virus DNA as Prognostic Indicators in Patients with Nasopharyngeal Carcinoma: A Prospective Study. Cancer Res Treat 2018, 50(3):701-711.

27. Lam WKJ, Chan KCA, Lo YMD. Plasma Epstein-Barr virus DNA as an archetypal circulating tumour DNA marker. J Pathol 2019, 247(5):641-649.

28. Xie X, Ren Y, Wang K, Yi B: Molecular Prognostic Value of Circulating Epstein-Barr Viral DNA in Nasopharyngeal Carcinoma: A Meta-Analysis of 27,235 Cases in the Endemic Area of Southeast Asia. Genet Test Mol Biomarkers 2019, 23(7):448-459.

29. Ou H, Huang Y, Zhao S, Zhou Y, Lv W. Prognostic value of Epstein-Barr virus DNA level for nasopharyngeal carcinoma: a meta-analysis of 8128 cases. Eur Arch Otorhinolaryngol 2020, 277(1):9-18.

30. Hsu CL, Chan SC, Chang KP, Lin TL, Lin CY, Hsieh CH, Huang SF, Tsang NM, Lee LY, Ng SH, et al. Clinical scenario of EBV DNA follow-up in patients of treated localized nasopharyngeal carcinoma. Oral Oncol 2013, 49(6):620-625.

31. Twu CW, Wang WY, Chen CC, Liang KL, Jiang RS, Wu CT, Shih YT, Lin PJ, Liu YC, Lin JC. Metronomic adjuvant chemotherapy improves treatment outcome in nasopharyngeal carcinoma patients with postradiation persistently detectable plasma Epstein-Barr virus deoxyribonucleic acid. Int J Radiat Oncol Biol Phys 2014, 89(1):21-29.

32. Chen FP, Huang XD, Lv JW, Wen DW, Zhou GQ, Lin L, Kou J, Wu CF, Chen Y, Zheng $Z Q$, et al. Prognostic potential of liquid biopsy tracking in the posttreatment surveillance of patients with nonmetastatic nasopharyngeal carcinoma. Cancer 2020.

33. Chang YS, Tyan YS, Liu ST, Tsai MS, Pao CC. Detection of Epstein-Barr virus DNA sequences in nasopharyngeal carcinoma cells by enzymatic DNA amplification. J Clin Microbiol 1990, 28(11):2398-2402.

34. Lo YM, Chan LY, Lo KW, Leung SF, Zhang J, Chan AT, Lee JC, Hjelm NM, Johnson PJ, Huang DP. Quantitative analysis of cell-free Epstein-Barr virus DNA in plasma of patients with nasopharyngeal carcinoma. Cancer Res 1999, 59(6):1188-1191.

35. Fournie GJ, Courtin JP, Laval F, Chale JJ, Pourrat JP, Pujazon MC, Lauque D, Carles P. Plasma DNA as a marker of cancerous cell death. Investigations in patients suffering from lung cancer and in nude mice bearing human tumours. Cancer Lett 1995, 91(2):221-227. 